\title{
Burn Patterns and Seasonal Change at a Specialized Burn Facility -5 years review
}

Mata Ribeiro, Luís. Vieira, L. Guerra, A.

Hospital São José, Plastic Surgery Department - Lisbon, Portugal

\section{Introduction:}

In Portugal there are many burn victims, some of them having to be admitted at dedicated Burn Centres. This leads to great social and economic costs. Even though several studies investigated the impact of temporal factors on the incidence of trauma admissions, hardly any data exists concerning the impact of seasonal change on burn profile. The main purpose of this study is trying to understand the real importance of the changing seasons on burn patients.

\section{Methodology:}

This retrospective analysis included all burn patients ( $\geq 18$ years; 420 patients) admitted to the Burn Unit at Hospital São José (Lisboa) between December 2010 and December 2015 (5 years). Data was extracted from medical records and included etiology, depth and extension of burn. This information was then grouped in 4 temporal categories: Summer, Autumn, Winter,
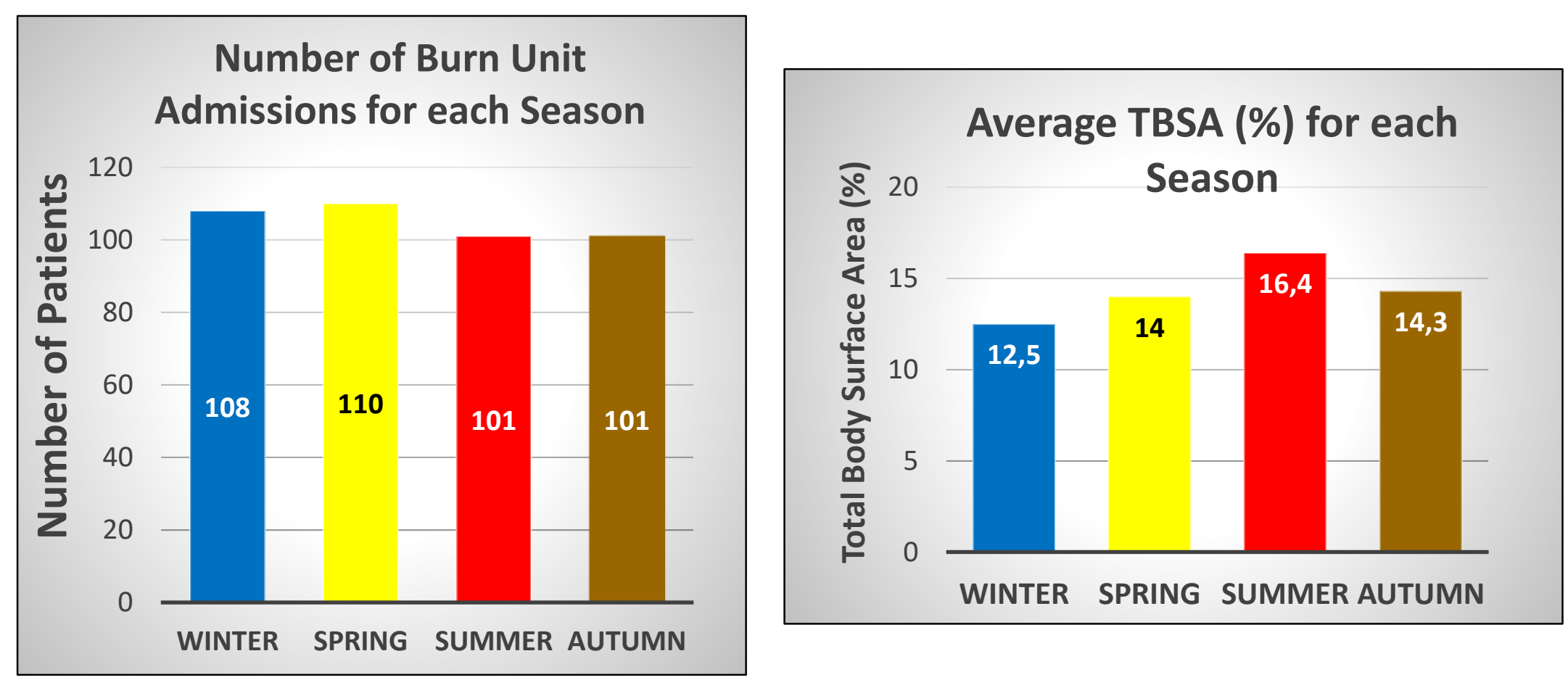

Spring.

\section{Results:}

The number of patients admitted to this Burn Unit did not show seasonal variation. Winter seems to be associated with greater depths of burn but with shorter TBSA and length of stay. The highest TBSA happened during Summer. Fire burns were less predominant during Spring.
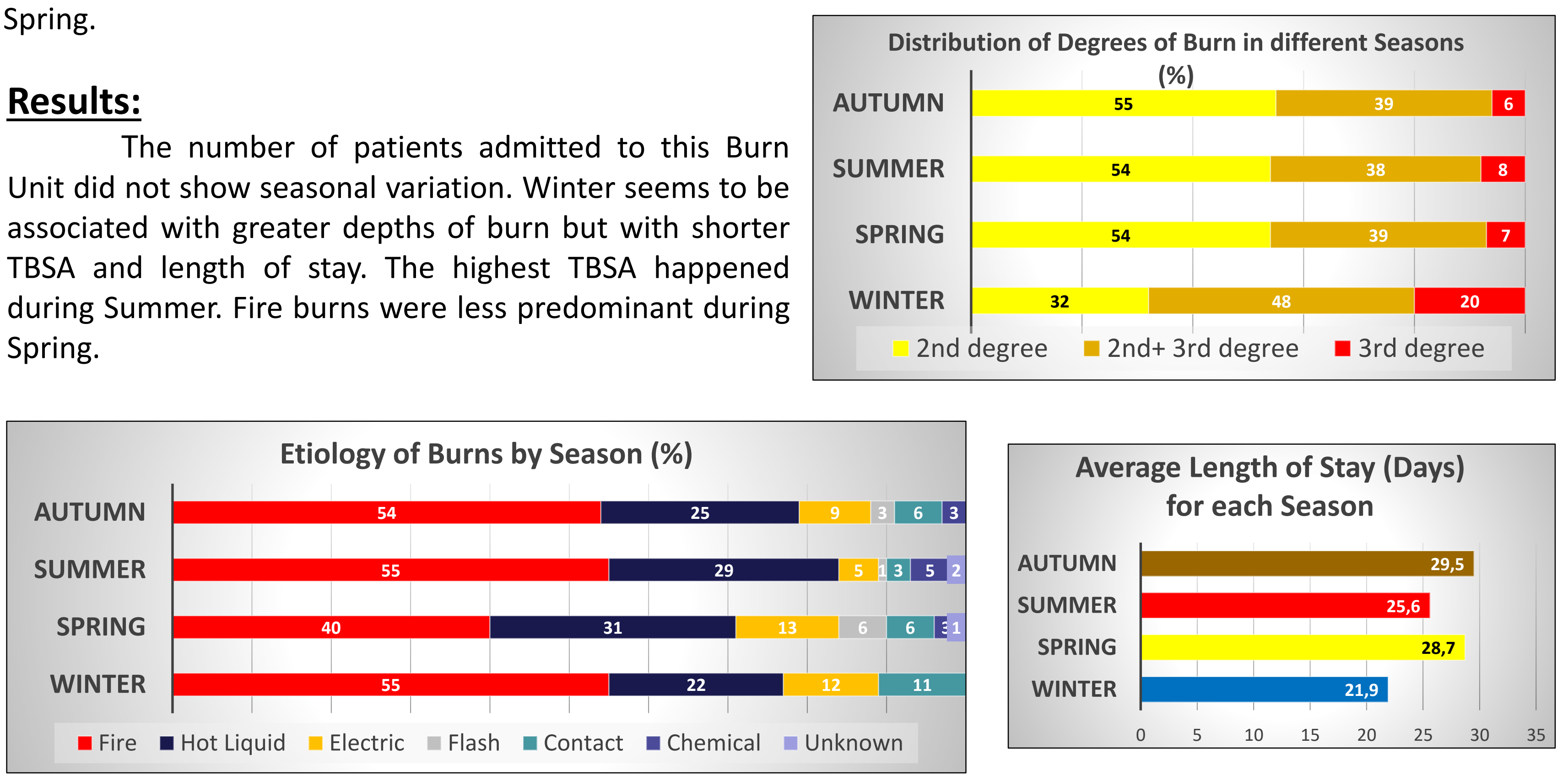

\section{Conclusion:}

Although the number of admissions was very similar, there were several differences on the burn profiles between seasons. The number of burns in Portugal is still very high and preventive measures are sometimes ineffective. It is essential to anticipate all these variables to delineate more effectively the preventive strategy. 